This is a preprint version of the paper:

The final publication is available at Springer via http://dx.doi.org/10.1631/FITEE.1900549

\title{
Interactive Visual Labelling versus Active Learning: An Experimental Comparison
}

\author{
Mohammad Chegini ${ }^{\dagger 1,2}$, Jürgen Bernard ${ }^{3}$, Jian Cui $^{2}$, Fatemeh Chegini ${ }^{4}$, \\ Alexei Sourin ${ }^{2}$, Keith Andrews ${ }^{1}$, Tobias Schreck ${ }^{1}$ \\ ${ }^{1}$ Graz University of Technology, Graz, Austria \\ ${ }^{2}$ Nanyang Technological University, Singapore \\ ${ }^{3}$ University of British Columbia, Vancouver, Canada \\ ${ }_{4}^{4}$ ax Planck Institute for Meteorology, Hamburg, Germany
}

\begin{abstract}
Methods from supervised machine learning allow the classification of new data automatically and are tremendously helpful for data analysis. The quality of supervised learning depends not only on the type of algorithm used but, importantly, also on the quality of the labelled dataset used to train the classifier. Labelling instances in a training dataset is often done manually, relying on selections and annotations by expert analysts and is often a tedious and time-consuming process.

Active learning algorithms can automatically determine a subset of data instances for which labels would provide useful input to the learning process. Interactive visual labelling techniques are a promising alternative, providing effective visual overviews from which an analyst can simultaneously explore data records and select items to a label. By putting the analyst in the loop, higher accuracy can be achieved in the resulting classifier. While initial results of interactive visual labelling techniques are promising in the sense that user labelling can improve supervised learning, many aspects of these techniques are still largely unexplored.

This paper presents a study conducted using the mVis tool to compare three interactive visualisations (similarity map, SPLOM with scatterplot, and parallel coordinates) with each other and with active learning for the purpose of labelling a multivariate dataset. The results show that all three interactive visual labelling techniques surpass active learning algorithms in terms of classifier accuracy and that users subjectively prefer the similarity map over SPLOM with scatterplot and parallel coordinates for labelling. Users also employed different labelling strategies depending on the visualisation being used.
\end{abstract}

Key words: Interactive Visual Labelling; Active Learning; Visual Analytics https://doi.org/10.1631/FITEE.1900549

CLC number: TP311

\section{Introduction}

Labelling is assigning a class from the label alphabet to an instance (a record) in a multivariate dataset. Supervised machine learning algorithms, such as classifiers (Bishop, 2006), must be trained

\footnotetext{
$\ddagger$ Corresponding author

(1) ORCID: Mohammad Chegini, https://orcid.org/0000-00023516-8685

(C) Zhejiang University and Springer-Verlag GmbH Germany, part of Springer Nature 2020
}

on a labelled dataset in order to perform. These methods learn how to generalise new data, based on existing known data examples which are provided with a class label. Creating a training dataset is essential to find a small subset of a dataset that delivers the best accuracy for the classifier. Although labelling a dataset is necessary, it can be a dull, timeconsuming, and expensive task.

To address this problem, active learning algorithms can help the analyst by suggesting instances 


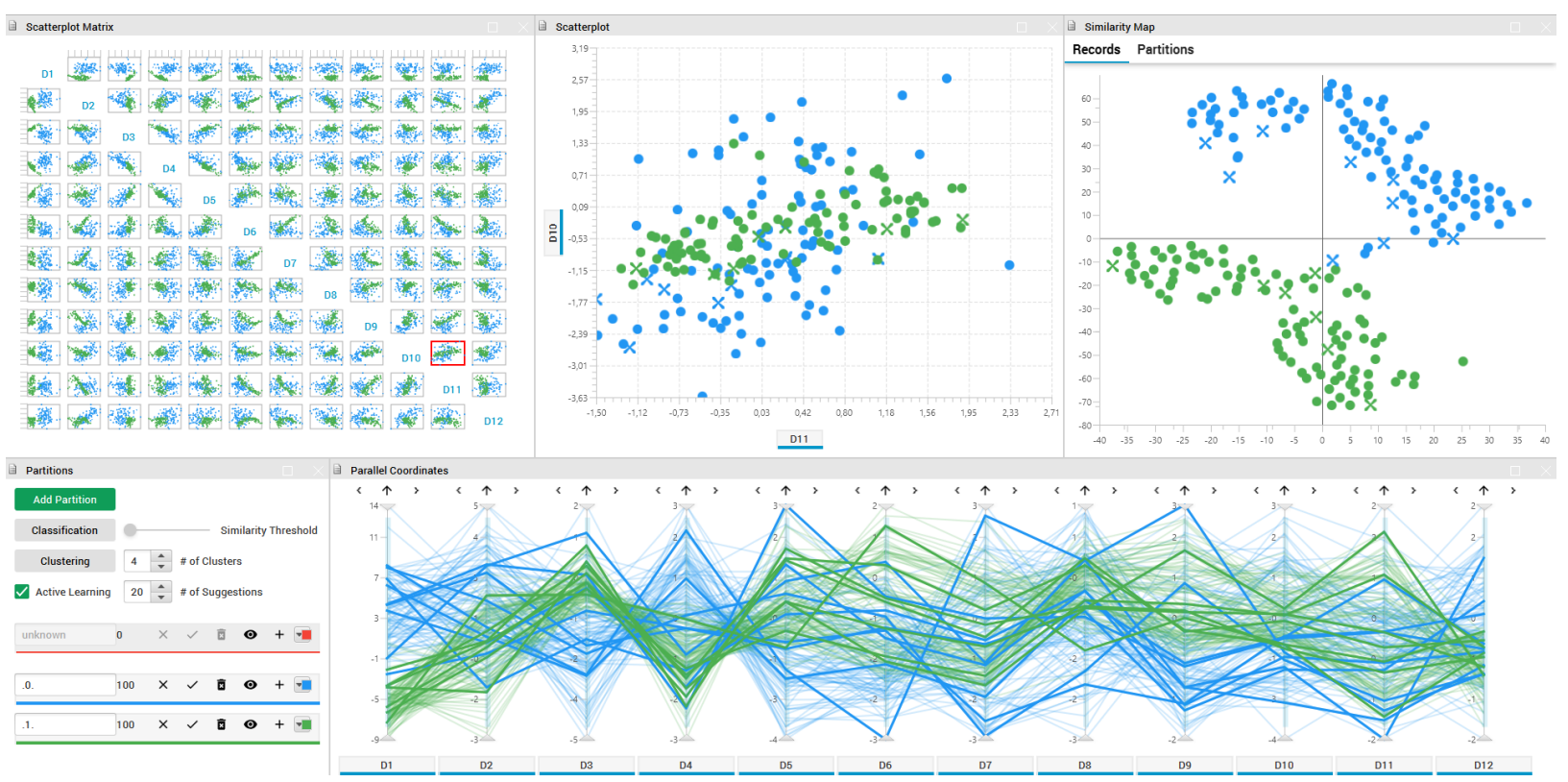

Fig. 1: The mVis tool (Chegini et al. 2019a), showing the SPLOM at top left, detailed scatterplot top middle, similarity map top right, and parallel coordinates bottom right, for the MNIST2 dataset. The partitions panel at bottom left shows the currently defined classes (label alphabet). Instances are colour-coded by class, here green for 1s and blue for 0s. Instances with confirmed labels are shown as crosses in the scatterplots and similarity map and as thick lines in the parallel coordinates. Suggestions from the classifier are shown as solid circles in the scatterplots and similarity map and as thin lines in the parallel coordinates.

to label (Settles, 2009). Active learning algorithms effectively reduce the number of records which need to be interactively labelled. Active learning techniques require heuristics for record selection, which often depend on the classification problem or data characteristics. Furthermore, interactive visual labelling (VIAL) (Bernard et al., 2018c ) tools build explorable visual overviews on top of active learning algorithms and can outperform classic active learning techniques in term of accuracy (Bernard et al. 2018a). Such combined tools allow an analyst to label a multivariate dataset in a visual environment, while receiving feedback and guidance from the system. Based on the overall data characteristics perceived by the analyst, conscious choices can be made as to what distinguishes groups of data and how many groups there should be, and representative records can be labelled. Immediate feedback can be given regarding the current set of labelled records, for example by visualising changes and improvements to the given classifier in response to given changes in labelling. Thereby, users can also gain an understanding of which choices affect the classifiers, and hence contribute to understandable and explainable machine learning models.

Since there are multiple visualisation and interaction techniques, the following research question arises: How do characteristics of these techniques and datasets affect performance and user experience for visual interactive labelling tasks? This key question will be broken down into several sub-questions in Section 4.1. To address them, this paper describes a comparative user study of three well-known interactive visualisation techniques for visual labelling: similarity map, scatterplot matrix (SPLOM), and parallel coordinates (Inselberg, 1985). Using the existing $\mathrm{mVis}$ visual data exploration tool (Chegini et al., 2019a), nine machine learning experts labelled two multivariate datasets in each of these three views separately. The quantitative measures from these tasks are accumulated and compared to each other and to active learning algorithms. In addition, the techniques are compared to each other in terms of user experience. The results confirm that involving the user in labelling using visual exploration facilities can improve the machine learning process and enhance model understanding. 


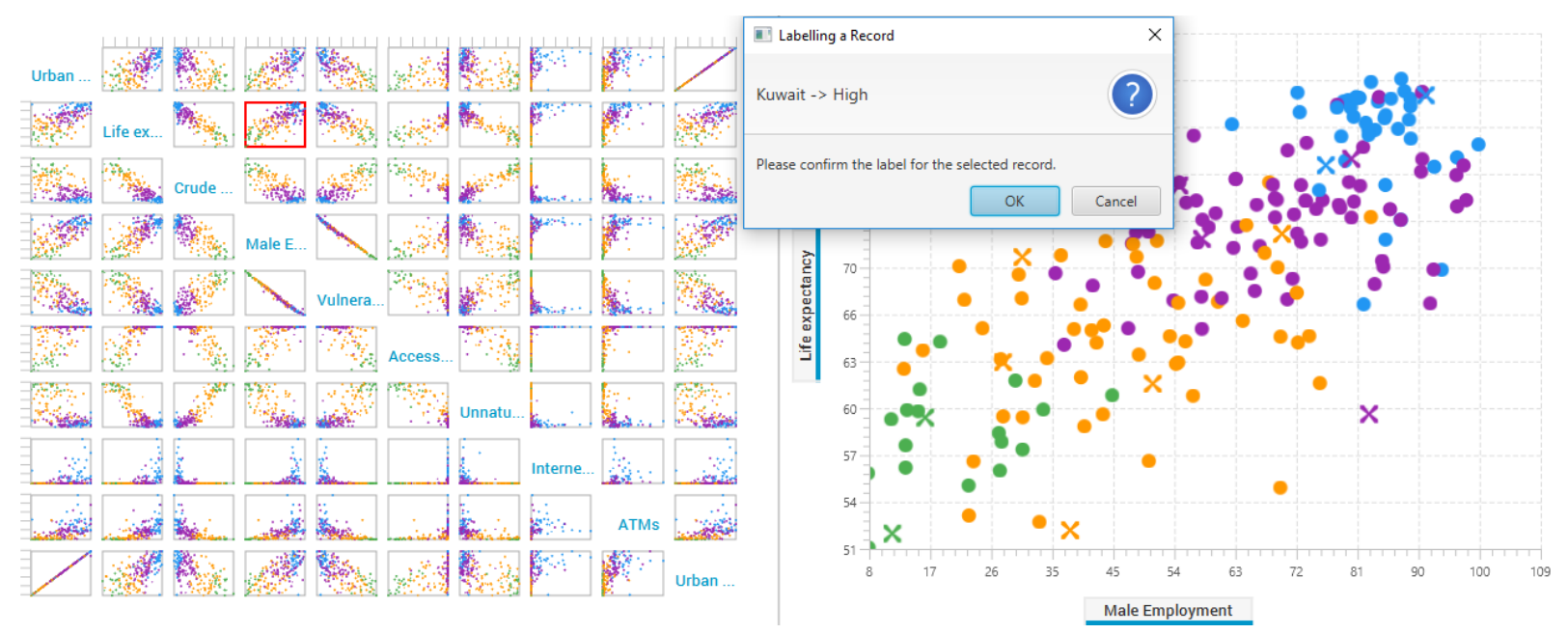

Fig. 2: The SPLOM with scatterplot visualisation of the WB dataset, as used by a test participant. Instances are colour-coded by class. Instances with confirmed labels are shown as crosses, suggestions from the classifier are shown as solid circles. The user has selected the scatterplot of Life Expectancy versus Male Employment in the SPLOM on the left and has selected the instance of Kuwait for labelling in the detailed scatterplot view on the right. The dialogue on the upper middle of the screen asks the user to confirm the label for that instance.

\section{Related Work}

Semi-supervised machine learning algorithms require some initial labelled instances (data records), and later the system acquires further labelled instances with the help of the oracle (i.e., analyst). Active learning (AL) strategies provide guidance by asking the analyst to label those instances which might provide better differentiation. Common active learning strategies include Smallest Margin (Scheffer et al., 2001, Wu et al., 2006), Entropy-Based Sampling (Settles and Craven, 2008), and Least Significant Confidence (Culotta and McCallum, 2005). These three strategies are fast, and are commonly used as uncertainly sampling active learning strategies (Bernard et al., 2018b). For the robustness of the experiment, in this paper, all three techniques are included in the comparison with interactive visual techniques.

In contrast, supervised machine learning algorithms require a sufficient number of labelled instances at the beginning. Classification techniques, such as Random Forest (Tin Kam Ho, 1995), are among these algorithms. Classifiers are an essential part of both active learning and interactive visual labelling strategies. Classifiers are used to provide visual feedback to the user during interactive labelling.
In this paper, in order to remove any potential bias, Random Forest is used as the classification technique for both the active learning and the visual interactive labelling techniques.

To date, multiple strategies for interactive visual labelling have been proposed. For example, Heimerl et al. (2012) incorporates active learning for interactive visual labelling of text documents. Höferlin et al. (2012) introduced inter-active learning, which extends active learning to a visual analytics process for building ad-hoc training classifiers. Bernard et al. (2018c) proposed Interactive Visual Labelling (VIAL), a unified process combining model-based AL strategies with visual analytics techniques. Interactive visual labelling strategies integrate various machine learning and visual analytics strategies to label an unlabelled dataset so it can be used to train a machine learning model. Bernard et al. (2018a) ran an experiment to show interactive visual labelling strategies can outperform pure active learning algorithms in terms of performance and accuracy. Later, Chegini et al. (2019a) integrated interactive labelling into $\mathrm{mVis}$, a tool built based on previous work by Shao et al. (2017) and Chegini et al. (2018). mVis provides visual analysis of high-dimensional data using multiple coordinated views, including similarity maps, SPLOM, and parallel coordinates. mVis' in- 


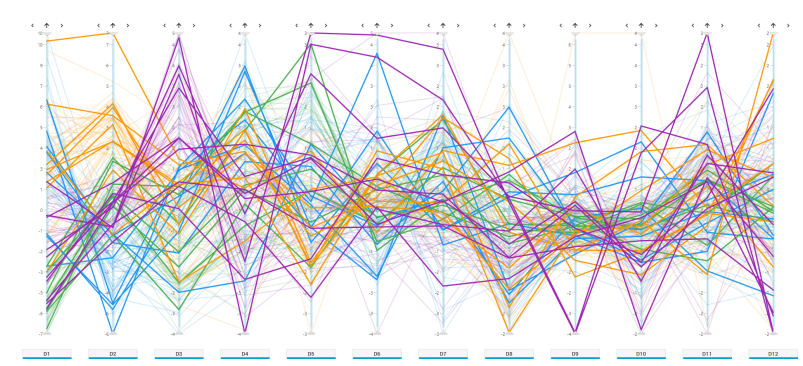

(a) MNIST4.

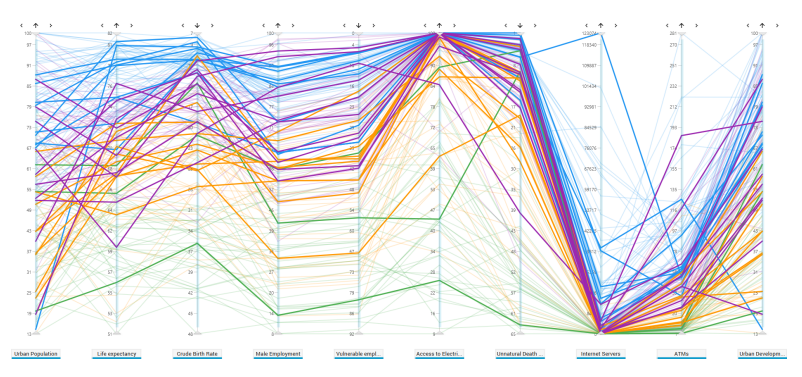

(b) WB.

Fig. 3: Parallel coordinates visualisations of (a) the MNIST4 and (b) the WB datasets. Instances are colourcoded by class. Instances with confirmed labels are shown as thick lines, suggestions from the classifier are shown as thin lines.

teractive labelling functionality allows users to create and name groups (classes) and add instances to them. Filtering and colour-coding support efficient comparison of the labelled groups across the different views. In a preliminary study (Chegini et al. 2019b), $\mathrm{mVis}$ was found to be intuitive and usable, helping analysts to gain insight into their data, and hence provides a sound technical basis for the comparative study.

\section{Methods}

Using $\mathrm{mVis}$, the performance of three different visualisation techniques for labelling a multivariate dataset was compared. Figure 1 shows $\mathrm{mVis}$ with a two-class subset of the MNIST dataset (LeCun et al. 1998). Since prior studies have shown that users prefer t-SNE over PCA and MDS for interactive visual labelling, t-SNE (Maaten and Hinton, 2008) algorithm is used for the similarity map.

For the SPLOM, all bivariate combination are shown in a matrix, and the user can select any of them to examine more closely in the scatterplot view. In the Parallel Coordinates view, the analyst can rearrange or invert dimensions and filter out records.

In general use, $\mathrm{mV}$ is allows the analyst to select one or multiple instances for labelling. Every time a set of instances is labelled, the Weka implementation of a Random Forest Classifier (Hall et al., 2009) runs in the background and suggests potential labels for all currently unlabelled instances by colour-coding according to their suggested class. Instances whose labels have been confirmed by the user are made visually distinct from instances with labels suggested by the classifier. Confirmed instances are shown as crosses in the scatterplots and similarity map and as thick lines in the parallel coordinates. Suggestions are shown as solid circles in the scatterplots and similarity map and as thin lines in the parallel coordinates. For the experiment described in this paper, the user was restricted to selecting a single instance at each step, which was then assigned its pre-assigned class.

Later, in order to assess the classification performance of the interactive visual labelling techniques, three methods were used: active learning, greedy selection, and random selection. Three active learning methods were used: Smallest Margin, EntropyBased Sampling, and Least Significant Confidence and the average accuracy in each step was used to compare the results. For the greedy method, the classifier was run for all possible instances for labelling, and the one with the best accuracy was selected. Greedy selection represents the best possible labelling result, and is the theoretical upper limit of what could be achieved by any visual labelling technique or active learning strategy. The random selection of instances was run 200 times, and the average accuracy in each step was used to compare the results. Random selection represents a practical lower limit for the accuracy a classifier should achieve.

The work of Bernard et al. (2018a) was chosen to describe the strategies of users for selecting labelling candidates. There, selection strategies were first grouped into data-centred and model-centred strategies. Data-centred strategies focus on the characteristics of data instances and include Dense Areas First, Centroid First, Equal Spread, Cluster Borders, Outliers, and Ideal Label. Model-centred strategies rely on visual feedback of the current state of the 
classification model and include Class Distribution Minimisation, Class Borders, Class Intersection, and Class Outliers. In addition to the strategies defined by Bernard et al. (2018a), in this study, another strategy was observed, which was named Visual Centre. Here, users would select instances in the centre of the visualisation they were currently focussed on.

\section{Study Design}

A comparative experiment was conducted to evaluate the effectiveness of three individual visualisation techniques for interactive labelling, based on which records were selected by test users for labelling. The three techniques were similarity map, SPLOM with scatterplot for a detailed view, and parallel coordinates. The comparison was both quantitative and qualitative.

\subsection{Research Questions}

The study addressed three main research questions:

$R Q_{1}$ How do three individual visualisation techniques, (similarity map, SPLOM, and parallel coordinates) compare in terms of accuracy of the resulting classifier?

$R Q_{2}$ How does interactive visual labelling (IVL) with the three visualisation techniques compare to non-interactive labelling based on active learning (AL) selection?

$R Q_{3}$ Which of the three visualisation techniques are rated higher by users in terms of user experience and confidence during selection of records to label?

$R Q_{4}$ Do users adopt different labelling strategy depending on the visualisation being used?

The user was asked to choose 30 instances for labelling, one instance at a time, each of which was then labelled with its (correct) pre-assigned label from the ground truth.

Regarding $R Q_{1}$, the accuracy of the classifier was computed after each time an instance had been chosen for labelling, using the current training set (i.e. the set of records with confirmed labels at a particular point in time). The accuracy is simply the number of correct predictions divided by the

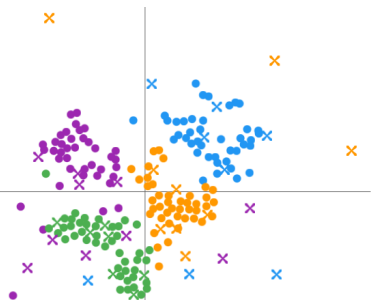

(a) MNIST4.

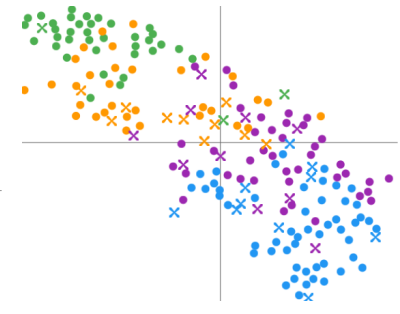

(b) WB.
Fig. 4: Similarity maps of (a) the MNIST4 and (b) the WB datasets. Colours indicate classes. Instances with confirmed labels are shown as crosses, suggestions by the classifier are shown as solid circles.

total number of predictions. This experiment was concerned with which instances users chose to label, not with the actual labels which were then assigned. Hence, users were not actually asked to assign a label, simply to confirm the correct label from the ground truth (see Figure 2). To this end, after a user had chosen an instance to label, a pop-up window appeared showing the (pre-assigned) label for that instance, and was simply asked for confirmation. Once the label had been confirmed, the classifier ran in the background to refresh suggested labels for currently labelled instances. Participants were provided neither with guidance nor with any active learning suggestions about which instance to label next, but were asked to choose freely, and without time constraints. Participants were also not informed about the accuracy of the model as they worked, but they were shown a chart about accuracy after they had finished working with each dataset.

Regarding $R Q_{2}$, the three active learning algorithms were run for each dataset, the accuracy of the resulting classifier was calculated for each step, and then averaged over all three AL algorithms. This provided the baseline for comparison. The ratings for $R Q_{3}$ were collected after the three visualisation had been for each dataset. The labelling strategies used by each user for $R Q_{4}$ were determined by analysing the thinking aloud protocol, screen recording, and interview responses.

\subsection{Datasets}

Three datasets were used in this study. The first dataset is a two-class subset of the classic MNIST dataset (LeCun et al. 1998), comprising images of hand-written digits in one of two classes: $0 \mathrm{~s}$ and 
1s. It was used to explain $\mathrm{mVis}$ to the participants in the tutorials phase of east test session. The 784 dimensions of the original dataset were reduced to 12 by PCA (Jolliffe, 2002) and named D1 through D12. The test dataset comprised 200 records with 100 records in each class. This dataset will be referred to as the MNIST2 dataset and is shown in Figure 1.

The second dataset is an MNIST dataset with 50 records in each of four classes (200 records total), representing the digits $0,1,6$, and 7 . Like the first dataset, this dataset was reduced to 12 dimensions with PCA. This dataset will be referred to as the MNIST4 dataset. Figure 3a and Figure 4a show this dataset in parallel coordinates and a similarity map.

The third dataset is a socio-economic statistical dataset published by the World Bank (WB, 2019). Each record is a country. The ten dimensions represent attributes such as Urban Population, Life Expectancy, and Access to Electricity. The 192 records (countries) are classified (unevenly) into one of four economic classes: lower income, lower-middle income, upper-middle income, and high income. This dataset will be referred to as the WB dataset. Figure 2, Figure $3 \mathrm{~b}$, and Figure $4 \mathrm{~b}$ show this dataset in SPLOM with scatterplot, parallel coordinates, and a similarity map.

\subsection{Participants and Setup}

The study was carried out in a quiet lab. Ten participants were initially recruited for the study, but one was later eliminated from the analysis due to technical problems. Of the nine remaining participants, three were female and six were male, with a median age of 29 years. All participants were familiar with machine learning and scatterplot visualisations. Two-thirds (6 of 9 ) were familiar with SPLOM and parallel coordinates. Two-thirds (a different 6 of 9) had previous experience in labelling multivariate datasets.

During their test session, participants were asked to think aloud, and to ask questions if they experienced any difficulties. At the end of the session, participants were encouraged to make suggestions for improvement. On average, each session lasted around 55 minutes, with the shortest and longest being 43 and 78 minutes, respectively. All sessions were captured by screen recording, and three sessions were additionally recorded with an external video camera for later analysis.

\subsection{Procedure and Tasks}

The test procedure with each participant comprised of four phases:

1. Opening: Introduction and background questionnaire.

2. Tutorial: Demonstration of $\mathrm{mVis}$ and practice with the MNIST2 dataset.

3. Test Session: Six experimental conditions, labelling each of the two datasets with each of the three visualisations.

4. Closing: Interview with the participant.

In the first phase, the facilitator explained the purpose of the study and the participants then filled out a background questionnaire. The questionnaire included four binary (yes/no) questions. In these questions, it was asked whether the participant had used machine learning algorithms, scatterplots, SPLOM, and parallel coordinates.

In the second phase, The facilitator first demonstrated the functionality of $\mathrm{mV}$ is with the MNIST2 dataset, explaining each of the three visualisation techniques and labelling two of the records. Then, users were asked to label a further 28 records by using all three visualisations.

In the third phase, each test user performed the labelling task for each of the two datasets (MNIST4 and WB) with each of the three visualisations (similarity map, SPLOM with scatterplot, and parallel coordinates). Each visualisation was maximised to full screen. The presentation order of these six experimental conditions was grouped by dataset but otherwise counterbalanced, as can be seen in Table 1. In each experimental condition, the test participant was asked to choose 30 instances for labelling (one after the other), which were then assigned their pre-assigned label (class). The experimental conditions were grouped by the dataset. One dataset was loaded, and labelling was completed with the three visualisations, then the second dataset was loaded for the final three visualisations. After each dataset had been explored with all three visualisations, test participants were asked to rate their experience and confidence in labelling the records for each visualisation: 


\begin{tabular}{|l|llllll|}
\hline & $C_{1}$ & $C_{2}$ & $C_{3}$ & $C_{4}$ & $C_{5}$ & $C_{6}$ \\
\hline$T P_{1}$ & M-S & M-X & M-P & W-S & W-X & W-P \\
$T P_{2}$ & M-S & M-P & M-X & W-S & W-P & W-X \\
$T P_{3}$ & M-X & M-S & M-P & W-X & W-S & W-P \\
$T P_{4}$ & W-P & W-S & W-X & M-P & M-S & M-X \\
$T P_{5}$ & W-P & W-X & W-S & M-P & M-X & M-S \\
$T P_{6}$ & W-X & W-P & W-S & M-X & M-P & M-S \\
$T P_{7}$ & M-X & M-P & M-S & W-X & W-P & W-S \\
$T P_{8}$ & W-X & W-S & W-P & M-X & M-S & M-P \\
$T P_{9}$ & M-P & M-S & M-X & W-P & W-S & W-X \\
\hline
\end{tabular}

Table 1: The presentation order of experimental conditions. Each row indicates a test participant and columns indicate the order of test conditions. The first letter indicates the dataset (M for MNIST4 and $\mathrm{W}$ for $\mathrm{WB})$. The second letter indicates the visualisation ( $\mathrm{S}$ for similarity map, $\mathrm{X}$ for SPLOM and scatterplot, and and $\mathrm{P}$ for parallel coordinates).

$Q_{1}$ From 1 to 5, how do you rate the labelling experience with \{visualisation technique\}?

$Q_{2}$ From 1 to 5 , how confident were you when selecting a new record with \{visualisation technique\}?

where 1 was the worst and 5 the best rating. In the $Q_{1}$, it was clarified to participants to rate the experience of interactive labelling and not the ease of the user interface or other aspects.

Finally, in the fourth phase, the facilitator interviewed the test participants about their experience and encouraged them to offer any feedback or suggestions they might have.

\section{Results}

The results of the study will be discussed for each of the three visualisation techniques (similarity map, SPLOM with scatterplot, and parallel coordinates) in terms of the four research questions from Section 4.1

\subsection{Similarity Map}

In terms of accuracy $\left(R Q_{1}\right)$, the similarity map outperformed SPLOM with scatterplot and parallel coordinates when using both the MNIST4 and WB datasets (see Figure 5).

Comparing with active learning $\left(R Q_{2}\right)$, the similarity map consistently outperforms active learning in both datasets, as can be seen in Figure 6 .

Regarding the ratings of users $\left(R Q_{3}\right)$, the sim- ilarity map was rated higher than the other two visualisation techniques, both in terms of labelling experience and selection confidence, as can be seen in Figure 7. Indeed, for labelling experience with the MNIST4 dataset, the mean rating of the similarity map was statistically significantly higher than the other two visualisations. All other differences in mean ratings were not statistically significant.

For both rating questions, the similarity map was rated slightly higher for the MNIST4 dataset than the WB dataset. This could be because the clusters in the MNIST4 dataset were more distinct and visible than those in the WB dataset, as shown in Figure $4 \mathrm{~b}$, This problem persists even when the projection algorithm for the similarity map is changed from t-SNE to PCA or MDS (Kruskal, 1964).

When using the similarity map, the strategies used by participants $\left(R Q_{4}\right)$ were similar to strategies observed during previous studies (Bernard et al. 2018a). In the similarity map, users tended to find distinct clusters from the beginning by using a Centroid First strategy. Therefore, the similarity map technique suffers less from the bootstrap problem (Figure 5). After identifying distinct clusters, users tried to find outliers and make clear borders. The second main strategy used by participants was Class Intersection, i.e. selecting records which are in the wrong visual section. These records are closer to a different cluster than their own. Based on the observations, identifying suspected incorrectly labelled records in a similarity map was found by the participants to be a rather well-defined task. Note that the accuracy of these labelling strategies depends on the quality of the similarity map, e.g., how faithfully distances in the high-dimensional data space are preserved in the $2 \mathrm{~d}$ projection space. An interesting variant for a future experiment would be to include measures for projection quality in the similarity map, for which different visualisation techniques exist (see, for example, Schreck et al. (2010)).

\subsection{SPLOM with Scatterplot}

Regarding the accuracy of the technique $\left(R Q_{1}\right)$, SPLOM with scatterplot performed slightly worse than similarity map with both datasets, but slightly better than parallel coordinates with the MNIST4 dataset and similar to parallel coordinates with the WB dataset (Figure 5). The advantage of SPLOM compared to similarity map and parallel coordinates 


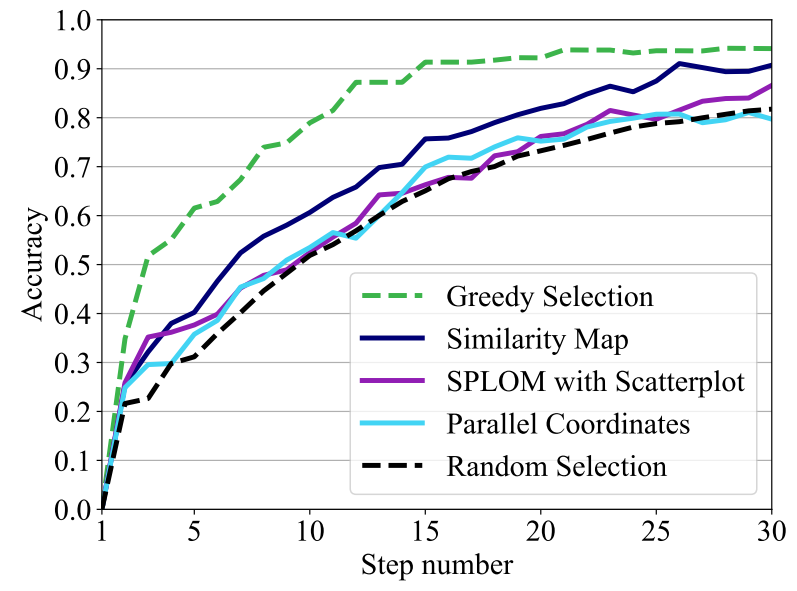

(a) Accuracy (MNIST4).

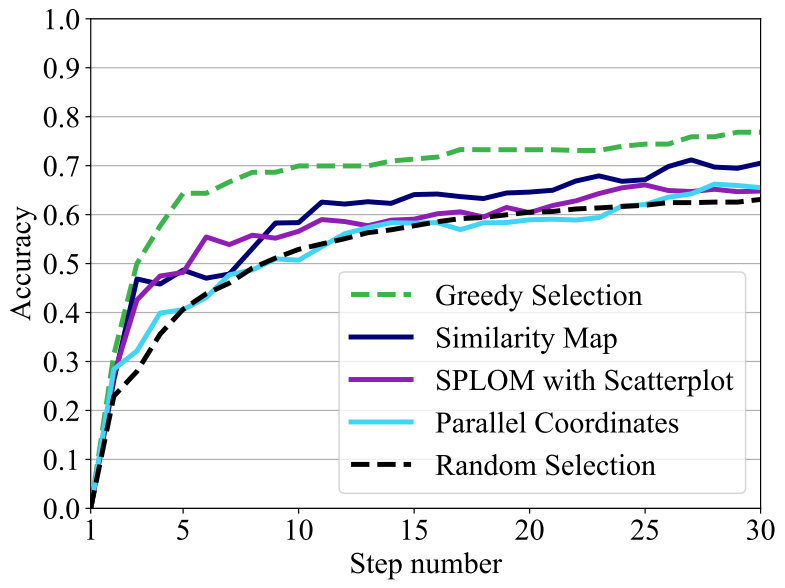

(b) Accuracy (WB).

Fig. 5: The accuracy of visual labelling depends on the interactive visualisation technique. The y-axis represents the accuracy, the $\mathrm{x}$-axis is the cumulative number of instances already labelled (step number). Greedy selection (green) represents a theoretical upper limit. Random selection (black) represents a practical lower limit.

was that it suffered less from the bootstrap problem.

SPLOM with scatterplot outperformed the active learning techniques $\left(R Q_{2}\right)$ for both datasets, as can be seen in Figure 6 .

Regarding the ratings of users $\left(R Q_{3}\right)$, the SPLOM with scatterplot technique was rated slightly lower than similarity map and slightly higher than parallel coordinates for both rating questions and with both datasets, as shown in Figure 7. However, the only statistically significant difference is the lower mean rating for labelling experience for SPLOM with scatterplot compared to similarity map with the MNIST4 dataset. Regardless of the ratings for both datasets being similar, users stated that selecting candidates with the WB dataset was easier, since the dimension names were semantically meaningful and therefore more understandable.

Regarding labelling strategy $\left(R Q_{4}\right)$ when using the SPLOM with scatterplot technique, users first attempted to find a scatterplot with well-spread records and then used the Centroid First strategy on this scatterplot. Later, some users selected scatterplots with well-separated clusters. Others preferred to select scatterplots which lacked well-separated clusters and attempted to separate them. In order to find outliers, some users tried brushing and linking. Most users tended to select a single scatterplot and continued to use it instead of changing to a differ- ent scatterplot. With the MNIST4 dataset, which lacks semantically meaningful dimensions, users selected a scatterplot with a clearer visual pattern, for example linear. Furthermore, users often selected scatterplots located in the centre of the SPLOM and ignored those in the outer reaches.

In general, users selected scatterplots from the SPLOM which: (a) have a specific pattern (for example, linear), (b) have well-separated classes, (c) have overlapping classes, (d) if the dimensions have semantically meaningful labels they select an interesting pair of dimensions based on the context, (e) randomly select scatterplots located in the centre of the SPLOM.

The disadvantage of the SPLOM with scatterplot technique is that it has many false positives. That is, clusters were not always visible and well separated, which confused some users. Moreover, the SPLOM technique was sometimes overwhelming for users.

\subsection{Parallel Coordinates}

Understanding parallel coordinates was hard for the users, mainly due to their lack of experience with this technique. Participants who were familiar with parallel coordinates performed better and were more confident during the experiment. Identifying patterns was difficult, particularly with the MNIST4 


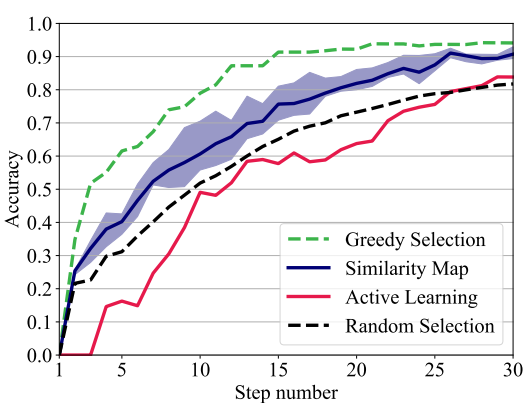

(a) Similarity map (MNIST4).

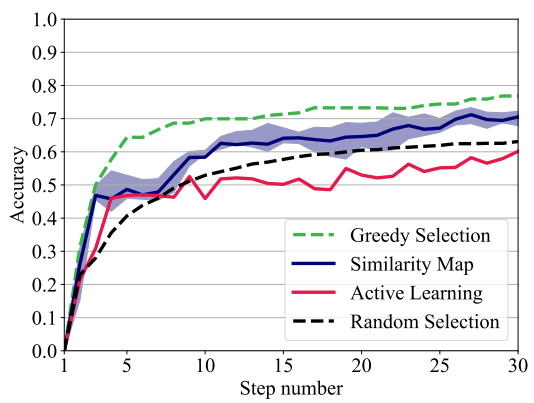

(d) Similarity map (WB).

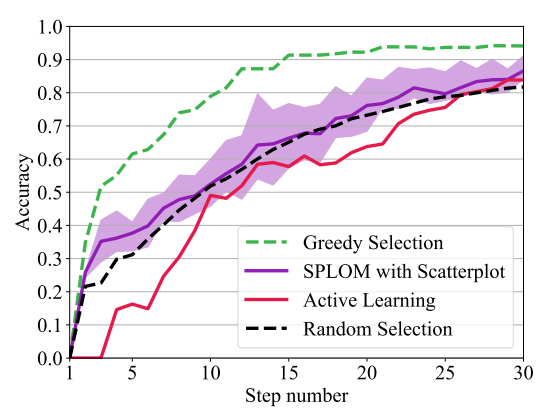

(b) SPLOM with scatterplot (MNIST4).

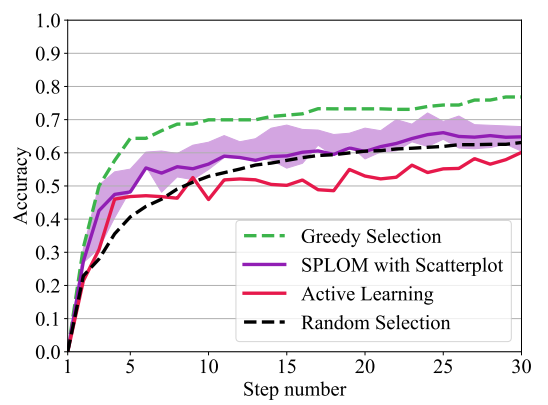

(e) SPLOM with scatterplot (WB).

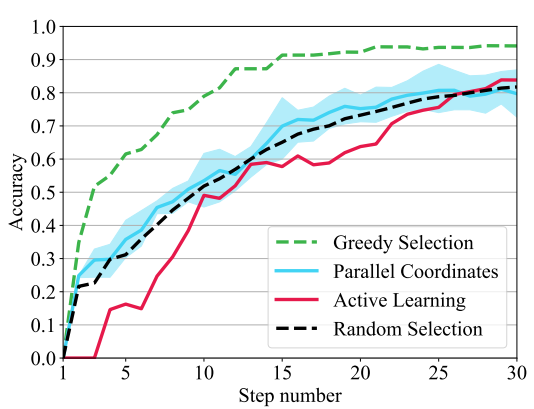

(c) Parallel coordinates (MNIST4).

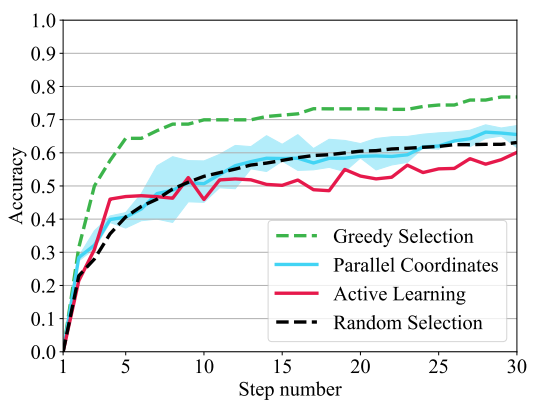

(f) Parallel coordinates (WB).

Fig. 6: Accuracy of the three interactive visual labelling techniques compared with active learning (red) for the MNIST4 and WB datasets. The semi-transparent coloured areas show the $25 \%$ and $75 \%$ quartiles.

dataset. Furthermore, parallel coordinates tended to be more cluttered, and therefore selections became more random over time. Some users were frustrated when they were forced to select points from parallel coordinates. One of the advantages of parallel coordinates was that it well guided the user's visual attention to extremes (peaks and valleys), enabling the users to identify these values easily. Furthermore, when users attempted to make borders for clusters in one single axis, using parallel coordinates was beneficial. On the other hand, one disadvantage of parallel coordinates was its lack of visual feedback, as stated by some users. Moreover, since users often focused on the centre of visualisation, the ordering of the axes was important when using parallel coordinates. Observations showed that if users rearranged the order of the axes, their experience could improve.

Regarding the accuracy of the classifier $\left(R Q_{1}\right)$, parallel coordinates performed about as poorly as SPLOM with scatterplot with the MNIST4 dataset and slightly worse than SPLOM with scatterplot with the WB dataset. Parallel coordinates also suffered from the bootstrap problem, due to the users' tendency to select extreme values (peak and valleys) in the beginning and ignore the middle records, which usually included lower-middle income and upper-middle income countries.

Parallel coordinates outperformed active learning $\left(R Q_{2}\right)$ in both datasets, although active learning catches up as more instances are labelled (see Figure 6).

Regarding user ratings $\left(R Q_{3}\right)$, parallel coordinates received the lowest ratings, both in terms of labelling experience and selection confidence for both datasets, as can be seen in Figure 7 The only statistically significant difference is the much lower mean rating for labelling experience for parallel coordinates compared to similarity map with the MNIST4 dataset. However, the mean can be misleading. Half of the users rated parallel coordinates 5 out of 5 when applied to the WB dataset, while the other half rated it poorly. The observations and interviews confirmed that some users strongly preferred parallel coordinates when the clusters were well separated, whilst others favoured other techniques.

In terms of labelling strategy $\left(R Q_{4}\right)$, partici- 


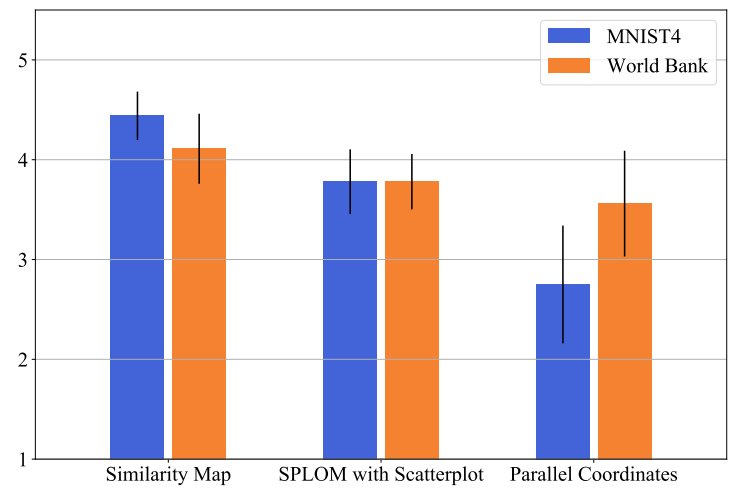

(a) Labelling experience.

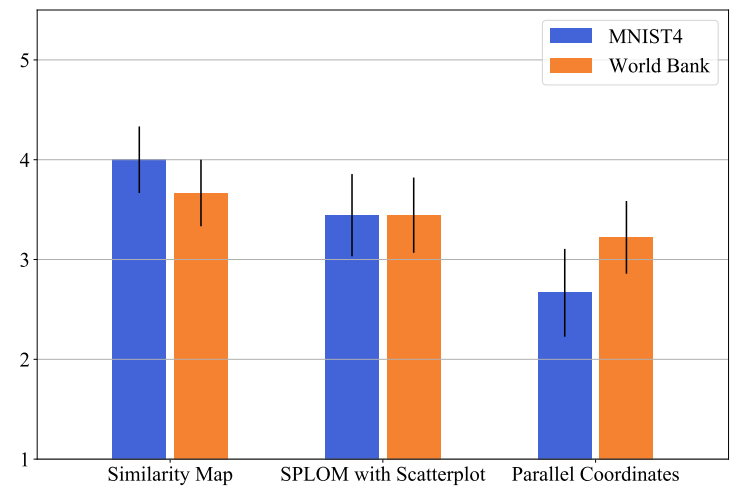

(b) Selection confidence.

Fig. 7: Mean ratings given by the test users for (a) labelling experience and (b) selection confidence for each of the three visualisations on a scale of 1 (worst) to 5 (best). Black lines represent standard error.

pants carried out the following strategies when using parallel coordinates: (a) selected records on a single axis based on their values, (b) focused on a combination of two axes, i.e., a line, (c) focused on the shape of the polyline or general picture in three or more axes, (d) focused on peaks and valleys, (d) randomly selected records on one axis or on a line between two axes. The users' main strategy was to select extreme values in an axis located in the centre of the visualisation. The Density First strategy was a common strategy used by the participants. At the beginning of the tasks, 60 per cent of the participants used the default order of the axes, and 40 per cent customised the order ( $\mathrm{mVis}$ allows to reorder axes interactively). Users rarely changed the order of the axes afterwards. When using parallel coordinates, users paid less attention to having an equal spread strategy, and therefore, the clusters were more imbalanced. Users also tried to identify class borders, but in the MNIST4 dataset finding such borders was difficult.

When using the parallel coordinates technique, some users occasionally became frustrated and selected random records located in the visual centre of the plots. A recurring problem was the users' tendency to selecting outliers, leading to the bootstrap problem, as can be seen in Figure 5. Furthermore, users selected higher values (peaks) more than lower (valley) values which lead to an imbalance in the selection of peaks and valleys. When using parallel coordinates, users deployed the Ideal Labels strategy more than when using other techniques.

\section{Discussion}

The results of the study are promising as they show that the classification performance of interactive visual labelling techniques can outperform those of active learning selection strategies. As shown in Figure 6, with the WB dataset, all three visualisations perform better after around 10 labelled instances than active learning. In contrast, with the MNIST4 dataset, active learning catches up with the interactive visual labelling techniques as the number of labelled instances increases.

Only a very limited number (9) of test users participated in this study. It would need to be repeated with a much larger number of test users, in order for the results to be more generalisable. The results were also obtained for very specific choices of visualisation and datasets, and their generalisation would require additional validation. Labelling a dataset can be a dull task. Three participants mentioned interactive visual labelling is enjoyable and feels like playing a game.

Some visualisations appear to be better suited to interactive visual labelling than others. The similarity map seems to be the preferred view for labelling. This can be attributed to the fact that the similarity map reduces data, gives an overview of the similarity relationship, and is less complex than SPLOM with scatterplot or parallel coordi- 
nates. However, it was observed that when some example labelling is already available, some users prefer to use SPLOM with scatterplot for a more detailed insight into the high-dimensional data space and for label selection. It was also observed that users who are familiar with parallel coordinates perform better and are more confident using it for label decision making.

Parallel coordinates and SPLOM are suitable for finding relationships between dimensions, identifying clusters, and exploring data to make sense of it. Visualisation of the labelled data in parallel coordinates could be improved. As the number of labelled instances increases, it can become overwhelming for the user to find the next instance to label. A problem found in all three visualisation techniques is that of false labelling. When an instance is close to a specific cluster, the user believes the instance belongs to that cluster and does not select it for labelling.

Regarding differences in the two datasets, it was observed that the MNIST4 dataset appeared very cluttered in the parallel coordinates visualisation, and patterns were difficult to discern. Therefore, the results for this test condition may have suffered. In the WB dataset, the dimensions had semantically meaningful names, and users felt more comfortable choosing the axes in the parallel coordinates visualisation and choosing a particular scatterplot from the SPLOM. For example, users often chose the Access to Electricity axis for labelling low income countries.

It is interesting to observe in Figure 6 that active learning often performs worse than random selection, at least in terms of the simple metric of model accuracy. However, this study only looked at the first 30 labelled instances and AL strategies often start poorly (bootstrap problem), but outperform random selection in later phases Attenberg and Provost, 2010, Kottke et al. 2017).

In terms of improvements, one user mentioned a lack of control over the arrangement of scatterplots within a SPLOM. Another user mentioned that parallel coordinates and SPLOM might be adapted to show the most "important" dimensions. Such an idea is presented in other work by using eyetracking (Chegini et al., 2019c). Active learning was also mentioned by a participant as an additional form of visual guidance (Ceneda et al., 2016) for visualisation techniques. Another participant was curious to see the accuracy of the classifier after the selection of every instance, together with the number of already labelled instances from each class.

\section{Limitations and Future Work}

While the findings of this study are interesting, they also depend on a number of choices made and require further analysis. For the experiments, a number of settings were fixed, which could be varied as well. Three specific visualisations (similarity map, SPLOM with scatterplot, and parallel coordinates) were chosen and these were used individually for the labelling task. Many visual analytics systems provide multiple linked views and dynamic brushing. Indeed, $\mathrm{mVis}$ provides these features too, but they were not used in this study in order to simplify its design. Multiple linked views and brushing could possibly mitigate some of the disadvantages of single techniques, and lead to a hierarchical selection strategy. For example, users might want to select a group of points as labelling candidates from the similarity view, and then switch to SPLOM or parallel coordinates for detailed selection and labelling. In future, support might be included for, say, automatic ordering of dimensions in parallel coordinates or arrangement of the plots in the SPLOM.

To compare classification performance, three different active learning algorithms (Smallest Margin, Entropy-Based Sampling, and Least Significant Confidence) were selected. While the selected algorithms are robust and applicable for different classifiers, the design space of active labelling is large and more comparisons could be made.

In the accuracy comparison experiments, it is assumed that the user always assigns the true (ground truth) label for a data point, once it has been identified for labelling. While this corresponds to the notion of a user being an "oracle" in active learning, labelling errors could also be considered in a future experiment. Users could be allowed to freely pick a label, or even introduce a new label during interactive visual labelling. This would increase experimental complexity, but allow even more realistic assessments.

In many practical situations, the type and number of labels are not known in advance, but are determined in an iterative process. Also, in many practical problems, high-dimensional data attributes are often complemented with additional metadata and 
background information. For example, countries like in the WB dataset could be presented as map views. Including visualisation of such additional data, and studying how it is used during the labelling process, would be an interesting experiment to do.

In future work, it would be interesting to study the dynamics of the labelling process. For example, are there learning effects during labelling, where the choice of labels changes over time? In the experiment described in this paper, the number of labels was fixed at 30. A future experiment could let the user decide when to stop the labelling process. To this end, feature and model space visualisations could be helpful for the user to assess when label saturation has been reached.

\section{Concluding Remarks}

This paper presented a study comparing three interactive visualisations with each other and with active learning for the purpose of labelling a multivariate dataset. The study also explored subjective user ratings for the three interactive visualisations and discussed the labelling strategies employed by users with each them.

All three interactive visualisations performed better than active learning algorithms, in terms of classification accuracy (assuming the user always assigns the correct label to a selected data instance). The similarity map performed better than both SPLOM with scatterplot and parallel coordinates in both the MNIST4 and WB datasets. Nevertheless, parallel coordinates and SPLOM with scatterplot are useful in their own right, especially for datasets where the dimensions have semantically meaningful names. The results support the view that a user-inthe-loop approach is beneficial for creating training datasets. Finally, the paper presented some ideas for future work and further studies.

\section{References}

Attenberg J, Provost F, 2010. Inactive learning?: Difficulties employing active learning in practice. SIGKDD Explorations Newsletter, 12(2):36-41. https://doi.org/10.1145/1964897.1964906

Bernard J, Hutter M, Zeppelzauer M, et al., 2018a. Comparing visual-interactive labeling with active learning: An experimental study. IEEE Transactions on Visualization and Computer Graphics (TVCG), 24(1):298-308. https://doi.org/10.1109/TVCG.2017.2744818

Bernard J, Zeppelzauer M, Lehmann M, et al., 2018b. Towards user-centered active learning algorithms. Com- puter Graphics Forum (CGF), 37(3):121-132.

https://doi.org/10.1111/cgf.13406

Bernard J, Zeppelzauer M, Sedlmair M, et al., 2018c. Vial: A unified process for visual interactive labeling. The Visual Computer, 34(9):1189-1207. https://doi.org/10.1007/s00371-018-1500-3

Bishop CM, 2006. Pattern Recognition and Machine Learning. Springer.

Ceneda D, Gschwandtner T, May T, et al., 2016. Characterizing guidance in visual analytics. IEEE Transactions on Visualization and Computer Graphics, 23(1):111120.

https://doi.org/10.1109/TVCG.2016.2598468

Chegini M, Shao L, Gregor R, et al., 2018. Interactive visual exploration of local patterns in large scatterplot spaces. Computer Graphics Forum (CGF), 37(3):99-109. https://doi.org/10.1111/cgf.13404

Chegini M, Bernard J, Berger P, et al., 2019a. Interactive labelling of a multivariate dataset for supervised machine learning using linked visualisations, clustering, and active learning. Visual Informatics, 3(1):9-17. https://doi.org/10.1016/j.visinf.2019.03.002

Chegini M, Bernard J, Shao L, et al., 2019b. mVis in the wild: Pre-study of an interactive visual machine learning system for labelling. Proc IEEE Vis 2019 Workshop on Evaluation of Interactive Visual Machine Learning Systems (EVIVA-ML), p.1012, http://eviva-ml.github.io/papers/sub1012.pdf

Chegini M, Sourin A, Andrews K, et al., 2019c. Eye-tracking based adaptive parallel coordinates. SIGGRAPH Asia Poster (SA'2019), p. 44. https://doi.org/10.1145/3355056.3364563

Culotta A, McCallum A, 2005. Reducing labeling effort for structured prediction tasks. National Conference on Artificial intelligence (AAAI), p.746-751.

Hall M, Frank E, Holmes G, et al., 2009. The weka data mining software: An update. ACM SIGKDD Explorations Newsletter, 11(1):10-18. https://doi.org/10.1145/1656274.1656278

Heimerl F, Koch S, Bosch H, et al., 2012. Visual classifier training for text document retrieval. IEEE Transactions on Visualization and Computer Graphics, 18(12):2839-2848. https://doi.org/10.1109/TVCG.2012.277

Höferlin B, Netzel R, Höferlin M, et al., 2012. Inter-active learning of ad-hoc classifiers for video visual analytics. Proc IEEE Conference on Visual Analytics Science and Technology (VAST), p.23-32. https://doi.org/10.1109/VAST.2012.6400492

Inselberg A, 1985. The plane with parallel coordinates. The Visual Computer, 1(2):69-91. https://doi.org/10.1007/BF01898350

Jolliffe I, 2002. Principal Component Analysis. Springer.

Kottke D, Calma A, Huseljic D, et al., 2017. Challenges of reliable, realistic and comparable active learning evaluation. Proc Interactive Adaptive Learning Workshop, p.1-14, http:/ /www.daniel.kottke.eu/2017/challenges-ofreliable-realistic-and-comparable-active-learningevaluation/ 
Kruskal JB, 1964. Multidimensional scaling by optimizing goodness of fit to a nonmetric hypothesis. Psychometrika, 29(1):1-27.

https://doi.org/10.1007/BF02289565

LeCun Y, Bottou L, Bengio Y, et al., 1998. Gradient-based learning applied to document recognition. Proceedings of the IEEE, 86(11):2278-2324.

https://doi.org/10.1109/5.726791

Maaten Lvd, Hinton G, 2008. Visualizing data using t-sne. Journal of Machine Learning Research, 9(Nov):25792605.

Scheffer T, Decomain C, Wrobel S, 2001. Active hidden markov models for information extraction. International Conference on Advances in Intelligent Data Analysis (IDA), p.309-318.

Schreck T, von Landesberger T, Bremm S, 2010. Techniques for precision-based visual analysis of projected data. Information Visualization, 9(3):181-193. https://doi.org/10.1057/ivs.2010.2

Settles B, Craven M, 2008. An analysis of active learning strategies for sequence labeling tasks. Empirical Methods in Natural Language Processing (EMNLP), p.1070-1079

Settles B, 2009. Active learning literature survey. 1648 . University of Wisconsin - Madison.

Shao L, Mahajan A, Schreck T, et al., 2017. Interactive regression lens for exploring scatter plots. Computer Graphics Forum (CGF), 36(3):157-166. https://doi.org/10.1111/cgf.13176

Tin Kam Ho, 1995. Random decision forests. Proc 3rd International Conference on Document Analysis and Recognition (ICDAR), 1:278-282.

https://doi.org/10.1109/ICDAR.1995.598994

WB, 2019. Countries and economies. World Bank.

Wu Y, Kozintsev I, Bouguet JY, et al., 2006. Sampling strategies for active learning in personal photo retrieval. IEEE International Conference on Multimedia and Expo (ICME), p.529-532.

https://doi.org/10.1109/ICME.2006.262442 